\title{
Estimating the annual rate of de novo multiple aneurysms: three statistical approaches
}

\author{
James Ju Yong Cheong, Narcyz Ghinea, B.Sc. (Hons), \\ AND JAMES M. VAN GELDER, M.D. \\ Department of Neurosurgery, South Western Sydney Area Health Service, University of New South \\ Wales, Sydney, Australia
}

\begin{abstract}
Object. Individuals with unruptured intracranial aneurysms experience a higher rate of rupture if their history includes another aneurysm that has previously bled. The authors used systematic review and metaregression to estimate the annual rate of development of second de novo aneurysms after subarachnoid hemorrhage.

Methods. This investigation included studies in which more than 300 patients with intracranial aneurysms were described, and in which the age of the patients and the proportion with multiple aneurysms were documented. Studies describing delayed follow-up angiography that was performed after treatment of aneurysms were also reviewed.

Twenty studies were included in a between-study analysis. The univariate odds ratio (OR) for multiple intracranial aneurysms per year of age was 1.085 (95\% confidence interval [CI] 1.015-1.165); this value was calculated using a hierarchical model for between-study heterogeneity. Five studies were included that provided age stratification. The estimated OR for multiple intracranial aneurysms per year was 1.011 (95\% CI 1.005-1.018). Four follow-up studies were available.

Conclusions. According to the three different approaches (study-level, patient-level, and follow-up analyses), the estimated annual rates of development of de novo aneurysms were $1.62 \%$ (95\% CI $0.28-3.59 \%$ ), $0.28 \%$ (95\% CI $0.12-0.49 \%$ ), and $0.92 \%$ (95\% CI $0.64-1.25 \%)$, respectively. The estimated annual rate of development of second de novo aneurysms ranged from 0.28 to $1.62 \%$.
\end{abstract}

KEY WORDS • intracranial aneurysm • de novo aneurysm • subarachnoid hemorrhage

The rate of rupture is higher in individuals with unruptured intracranial aneurysms if their history includes another lesion that has previously bled. ${ }^{11}$ The incidence of $\mathrm{SAH}$ and the prevalence of unruptured intracranial aneurysms increase with age. ${ }^{3,9,23,27}$ The most common cause of delayed recurrent SAH after aneurysm clipping is de novo lesions. ${ }^{39}$ There is not enough information about the prevalence of de novo lesions in the years after an aneurysm ruptures or about the value, if any, of screening investigations. We estimated the association between patient age and multiple intracranial aneurysms by using systematic review and metaregression. This association was used to estimate the rate at which new intracranial aneurysms develop in patients who have experienced a previous rupture.

\section{Theory of Aneurysm Development}

We propose the following simple model for aneurysm development. In a given population of patients an intracranial aneurysm will occur in some individuals, and a second lesion will subsequently develop in a proportion of these. The prevalence of multiple intracranial aneurysms

Abbreviations used in this paper: $\mathrm{CI}=$ confidence interval; $\mathrm{OR}=$ odds ratio; $\mathrm{SAH}=$ subarachnoid hemorrhage. among patients who initially had single lesions increases with age. The overall rate of de novo aneurysm formation can be estimated based on this association. Both individuals with a single and those with multiple lesions may experience an aneurysm rupture. The incidence of such ruptures will be moderately higher in individuals with multiple lesions. The rate of de novo aneurysm formation will be slightly overestimated if it is derived from patients who mostly present with aneurysm rupture.

\section{CLINICAL MATERIAL AND METHODS Systematic Review of Patient Records}

A systematic review was performed to estimate the effect of age on the probability of harboring multiple intracranial aneurysms. In general, we followed the published guidelines for metaanalysis of observational studies. ${ }^{35} \mathrm{To}$ meet the inclusion criteria, studies were required to have the following characteristics: 1) focus on cerebral aneurysms or SAH as the major topic; 2) state the mean or median age of the patients; 3 ) include at least 300 patients; 4) detail the numbers of patients with both single and multiple intracranial aneurysms; 5) provide information about the methods used for diagnosis of the aneurysms; and 6) not specifically select individuals of a particular age or with a particular anatomical location of aneurysm. 
In studies in which investigators focused on risk factors for multiple intracranial aneurysms, the inclusion criteria were relaxed to allow reports with a minimum of 200 patients. The minimum number of 300 patients was used in accordance with a previous review. ${ }^{31}$ When two studies reported overlapping populations, only the more recent study was used. Studies were not used if they included $\mathrm{SAH}$ without a confirmed presence of aneurysms.

The literature review was conducted using Medline and EMBASE searches and included works published between 1970 and February 2004. A manual search was performed for the 6 months preceding August 2004 in five major neurosurgical journals. The terms "intracranial aneurysm" and "cerebral aneurysm" were used as text words and MESH headings with appropriate subheadings. A further search was performed to link cerebral aneurysms and the following risk factors: age, hypertension, sex, smoking, ischemic heart disease, and family history. Textbooks, journal bibliographies, and conference proceedings were also searched. Language restrictions were not used and the authors of the studies were not contacted. The data extracted from the studies are listed in Table 1. When possible, age-stratified patient-level data for the prevalence of multiple aneurysms were extracted. Follow-up studies that described the rate of development for de novo intra- cranial aneurysms were also reviewed. To meet inclusion criteria, the studies were required to report the mean duration of follow-up review, the number of patients screened, and the number of patients in whom a de novo intracranial aneurysm developed. A baseline angiogram was required. Patients were excluded if the follow-up angiogram was obtained because of a new SAH.

\section{Statistical Analysis}

Statistical analysis was performed using Gibbs sampling and hierarchical Bayesian models with the aid of Winbugs software (version 1.4, www.mrc-bsu.cam.ac.uk/ bugs). These models can be obtained from the corresponding author. The de novo aneurysm rate was derived using three separate models: a study-level metaregression of the association between age and multiple aneurysms, a patient-level metaregression of the association between age and multiple lesions, and a metaanalysis of followup studies.

Study-Level Model. For the study-level model the following hierarchical metaregression was used:

$m_{i} \sim \operatorname{Binomial}\left(\pi_{i}, n_{i}\right)$

$\operatorname{logit}\left(\pi_{i}\right)=\beta_{0}+\beta_{1}\left(\right.$ age $\left._{i}-51.5\right)+\alpha_{i}+\beta_{2} x_{i}$,

TABLE 1

Literature review of selected studies in which multiple intracranial aneurysms were described*

\begin{tabular}{|c|c|c|c|c|c|c|c|c|c|c|c|}
\hline Authors \& Year & $\begin{array}{l}\text { Country, Year } \\
\text { Study Began }\end{array}$ & $\begin{array}{l}\text { Total } \mathrm{w} / \\
\text { Lesions }\end{array}$ & $\begin{array}{l}\text { Selection } \\
\text { Criteria } \dagger\end{array}$ & $\begin{array}{l}\% \mathrm{w} / \\
\text { MA }\end{array}$ & $\begin{array}{l}\% \mathrm{w} / \\
\mathrm{HT}\end{array}$ & $\begin{array}{c}\% \\
\text { Male }\end{array}$ & $\begin{array}{c}\% \mathrm{w} / 4 \mathrm{~V} \\
\text { Angio }\end{array}$ & $\begin{array}{l}\text { Age } \\
\text { (yrs) }\end{array}$ & $\begin{array}{l}\% \mathrm{w} / \\
\mathrm{SAH}\end{array}$ & $\begin{array}{l}\% \text { in } \\
\text { MCA }\end{array}$ & $\begin{array}{l}\% \text { in } \\
\text { PCA }\end{array}$ \\
\hline Andrews \& Spiegel, 1979 & US, 1970 & 212 & 1 & 29.20 & 33.00 & 42.00 & NS & 51.1 & NS & NS & NS \\
\hline Rasmussen, et al., 1980 & Denmark, 1972 & 851 & 1 & 13.70 & NS & 40.00 & 32.0 & 45.5 & 89.0 & 31.30 & NS \\
\hline Nishioka, et al., 1984 & US, 1981‡ & 568 & 8 & 31.50 & NS & 42.00 & NS & NS & 100.0 & NS & NS \\
\hline Ostergaard \& Hog, 1985 & Denmark, 1977 & 737 & 1 & 17.10 & 24.60 & 39.50 & 100.0 & NS & NS & NS & NS \\
\hline Pasqualin, et al., 1986 & Italy, 1976 & 309 & 5 & 14.20 & NS & 47.60 & NS & 52.0 & 100.0 & NS & NS \\
\hline Pinna, et al., 1988 & Italy, 1974 & 350 & 3 & 12.00 & NS & 47.70 & NS & 48.0 & 100.0 & NS & NS \\
\hline Schievink, et al., 1988 & Netherland, 1983 & 343 & 3 & 17.50 & NS & 37.40 & NS & 48.0 & 100.0 & NS & NS \\
\hline Seifert, et al., 1988 & Germany, 1982 & 403 & 4 & 8.90 & NS & 40.20 & 100.0 & 44.5 & 100.0 & 23.00 & 4.50 \\
\hline Inagawa, 1990 & Japan, 1984 & 481 & 3 & 28.30 & NS & 41.60 & 100.0 & 59.0 & 100.0 & 32.00 & 6.20 \\
\hline Kassell, et al., 1990 & international, 1981** & 3521 & 7 & 19.00 & 21.20 & 38.50 & 100.0 & 50.4 & 100.0 & 22.30 & 8.00 \\
\hline Rinne, et al., 1995 & Finland, 1984 & 1314 & 1 & 23.00 & 31.40 & 52.50 & 23.5 & 49.1 & 91.0 & 47.00 & 5.00 \\
\hline Orz, et al., 1997 & Japan, 1991 & 1558 & 6 & 13.90 & NS & 41.40 & 100.0 & 59.9 & 80.1 & 33.00 & 6.40 \\
\hline Molyneux, et al., 2002 & international, 1999\| & 2143 & 4 & 21.30 & NS & 37.30 & NS & 52.0 & 100.0 & NS & NS \\
\hline Kaminogo, et al., 2003 & Japan, 1993 & 2037 & 3 & 17.72 & NS & 31.57 & 100 & NS & 100 & 28.20 & 4.61 \\
\hline Ogilvy \& Carter, 2003 & US, 1995 & 481 & 2 & 18.92 & NS & NS & NS & 53 & NS & 28.00 & 11.00 \\
\hline Weibers, et al., 2003 & $\begin{array}{l}\text { international, } \\
\text { prosp cohort, } 1995 \S\end{array}$ & 4060 & 2 & 35.32 & 40.64 & 24.43 & 100.05 & 53.286 & 0.0 & 29.04 & 4.90 \\
\hline
\end{tabular}

* Age refers to mean or median age. Abbreviations: angio = angiography; HT = hypertension; ISUIAI = International Study of Unruptured Intracranial Aneurysms Investigators; MA = multiple aneurysms; MCA = middle cerebral artery; NS = not stated; PCA = posterior circulation artery; prosp = prospective; $4 \mathrm{~V}=$ four-vessel.

$\dagger$ Selection refers to the sample population definition for the study, as follows: 1, all patients with intracranial aneurysms; 2 , patients with unruptured intracranial aneurysms; 3, ruptured lesions (aneurysmal SAH); 4, surgically treated aneurysmal SAH; 5, patients with aneurysmal SAH and intracranial hematomas; 6, patients with surgically treated intracranial aneurysms; 7, patients with aneurysmal SAH who were admitted within 0 to 3 days of bleeding incident; and 8, conservatively managed aneurysmal SAH.

\$ Cooperative Study of Intracranial Aneurysms and Subarachnoid Hemorrhage.

$\S$ International Study of Unruptured Intracranial Aneurysms Investigators Aneurysm Trial.

|| International Subarachnoid Aneurysm Trial.

** International Cooperative Study on the Timing of Aneurysm Surgery. 
$\pi=\exp \left(\beta_{0}\right) /\left[1+\exp \left(\beta_{0}\right)\right]$

$\alpha_{i} \sim \operatorname{Normal}\left(0, s^{2}\right)$, and

rate $_{51.5}=\beta_{1} \pi_{51.1}$ (see Appendix).

In this regression, $m_{i}$ was the number of patients with multiple intracranial aneurysms in study $i, n_{i}$ was the number of patients in that study, $\pi_{i}$ was the estimated probability of multiple lesions given that an individual had at least one aneurysm, and $a g e_{i}$ was the mean age of patients in the study $i$. The mean age over all the studies was 51.5 years. The overall probability of multiple intracranial aneurysms at the mean age of 51.5 years for the 31 studies included in the analysis was expressed as $\pi_{51.5}$. The annual rate for the development of de novo aneurysms was calculated from this probability by using the relationship given. Noninformative, normally distributed priors were used for the regression coefficients $\beta_{0}, \beta_{1}$, and $\beta_{2}$. The $\alpha_{i}$ represented the effects specific to each study and was used to accommodate the heterogeneity among the reports. The study effects were modeled as normally distributed, with a mean of zero and variance $s^{2}$. A uniform prior distribution was used for the standard deviation. Potential confounding variables among the studies are represented by $x_{i}$.

Patient-Level Model. A similar model was used for the patient-level metaregression, except that the proportion of patients with multiple intracranial aneurysms was stratified according to age groups $(\mathrm{k})$ and the mean age was 55.8 years: $m_{i k} \sim \operatorname{Binomial}\left(\pi_{i k}, n_{i k}\right)$, and $\operatorname{logit}\left(\pi_{i k}\right)=\beta_{0}+$ $\beta_{1}\left(\right.$ age $\left._{i k}-55.8\right)+\alpha_{i}$.

Follow-Up Model. A nonhierarchical (fixed-effect) metaanalysis was used for the four follow-up studies: $m_{i}$ $\operatorname{Binomial}\left(\pi_{i}, n_{i}\right)$, and $\pi_{i}=1-\exp \left(-r y_{i}\right)$, where $r$ is the annual rate of de novo aneurysm formation and $y_{i}$ is the mean years of follow up for study $i$. A fixed-effect model was used after testing for heterogeneity by using a bootstrap sampling method. We assumed there was no heterogeneity and simulated 1000 sets of patients in four studies, which was equivalent to the original data. For each simulation the total number of observed de novo aneurysms was assigned randomly among the four studies according to the number of follow-up years in each study. The likelihood was derived from hierarchical models for each of the random simulations and compared with the observed likelihood derived from the original data.

\section{RESULTS}

\section{Studies Used for the Regression}

Twenty-three studies were included in the systematic review; these described a total of 24,050 patients, among whom $5253(21.8 \%)$ harbored multiple aneurysms (Table 1 ). The proportion of patients with multiple aneurysms in each study ranged from 2.5 to $35 \%$. The proportion of men in the studies ranged from 24 to $55 \%$, and the mean ages of patients in the studies ranged from 44.5 to 59 years. The proportion of patients with hypertension was described in 10 studies and ranged from 21 to $52 \%$. The anatomical locations of the multiple intracranial aneurysms were described in 10 studies; middle cerebral artery aneurysms accounted for between 22 and $47 \%$ of the lesions, and posterior circulation aneurysms accounted for 4.5 to $11 \%$. The proportion of patients who presented with SAH was $100 \%$ in 14 studies, between 50 and $100 \%$ in five studies, $0 \%$ in one study, and not stated in three studies. In 10 of the studies four-vessel angiography was performed in all patients, in 10 the proportion was not reported, and in three the proportion ranged from 24 to $86 \%$. Four of the reports described multicenter international studies with multiple selection criteria. The mean patient ages in these four studies ranged from 50 to 53 years and the proportion with multiple lesions ranged from 19 to $35 \%$. The ages and proportions of patients with multiple intracranial aneurysms in the multicenter studies were similar to those in the other studies.

\section{Risk Factors for Multiple Aneurysms}

In six studies risk factors for multiple aneurysms were investigated (Table 2). Female sex, hypertension, and smoking were reported frequently. In contrast, age was generally not detected as a risk factor for multiple lesions. Ellamushi, et al., ${ }^{7}$ reported that multiple intracranial aneurysms increased with age in women. A statistic to describe the relationship between age and multiple lesions could only be extracted from one study. Juvela ${ }^{13}$ reported a univariate OR of 1.02 per year $(1.00-1.05, \mathrm{p}=0.09)$.

\section{Study-Level Analysis}

Twenty studies that described the mean age of patients were examined for an association between age and multiple intracranial aneurysms (Table 3, Fig. 1). Three studies were available for the patient-level but not the study-level analysis. This was because the mean age for the whole group was not stated and could not be estimated sufficiently accurately. The univariate OR for multiple lesions per year of age was 1.085 (95\% CI 1.015-1.165) according to a hierarchical model for between-study heterogeneity. The overall probability of multiple intracranial aneurysms at the mean age of 51.5 years was $19.73 \%(95 \%$ CI $15.45-27.07 \%)$.

TABLE 2

Risk factors for multiple aneurysms in selected studies*

\begin{tabular}{|c|c|c|c|c|}
\hline \multirow[b]{2}{*}{ Authors \& Year } & \multicolumn{2}{|c|}{ Univariate Factors } & \multicolumn{2}{|c|}{ Multivariate Factors } \\
\hline & Positive & Negative $\dagger$ & Positive & Negative $\dagger$ \\
\hline $\begin{array}{l}\text { Andrews \& Spiegel, } \\
1979\end{array}$ & HT, F, age & & HT & $\mathrm{F}$, age \\
\hline $\begin{array}{l}\text { Ostergaard \& Hog, } \\
1985\end{array}$ & HT & age, $\mathrm{F}$ & $\mathrm{F}, \mathrm{HT}$ & age \\
\hline Inagawa, 1990 & $\mathrm{~F}$ & age & & \\
\hline Qureshi, et al., 1998 & $\mathrm{~F}, \mathrm{sm}$ & age, $\mathrm{HT}, \mathrm{FH}$ & $\mathrm{sm}, \mathrm{F}$ & age, $\mathrm{FH}$ \\
\hline Ellamushi, et al., & $\mathrm{HT}, \mathrm{sm}, \mathrm{F}$ & age & $\mathrm{F}, \mathrm{HT}, \mathrm{sm}$ & age \\
\hline
\end{tabular}

* Risk factors selected were female sex, hypertension, smoking, age, and family history of aneurysms. Abbreviations: $\mathrm{FH}=$ family history; $\mathrm{sm}=$ active smoking.

$\dagger$ Not detected or not statistically significant. 
TABLE 3

Study-level analysis of risk factors for multiple aneurysms*

\begin{tabular}{|c|c|c|}
\hline Confounding Factor & $\begin{array}{c}\text { OR for } \\
\text { Confounding Factor }\end{array}$ & OR for Age \\
\hline random study effects & & 1.085 (1.015-1.165 \\
\hline middle yr of study & $1.002(0.984-1.028)$ & $1.103(1.024-1.193$ \\
\hline $\begin{array}{l}\text { studies that examined } \\
\text { risk factors for MA }\end{array}$ & $2.214(1.122-3$. & $1.058(0.995-1.130$ \\
\hline $\begin{array}{l}\text { studies w/ 100\% } \\
\text { ruptured aneurysms }\end{array}$ & $0.736(0.367-1.253)$ & $1.062(0.990-1.140$ \\
\hline $\begin{array}{l}\text { studies w/ 100\% } \\
4 \mathrm{~V} \text { angiography }\end{array}$ & $0.854(0.450-1.516)$ & $1.085(1.005-1.173$ \\
\hline$\%$ male patients & $0.981(0.950-1.014)$ & $1.062(0.982-1.142$ \\
\hline studies from Japan & $0.336(0.055-1.010)$ & \\
\hline studies from & $1.493(0.639-2.912)$ & \\
\hline $\begin{array}{l}\text { US/international } \\
\text { studies from }\end{array}$ & $2.102(0.967-4.422)$ & $1.191(1.053-1.352$ \\
\hline $\begin{array}{l}\text { Scandinavia } \\
\text { studies from Europe }\end{array}$ & 1 (comparison) & \\
\hline
\end{tabular}

* Numbers in parentheses represent CIs.

Potential confounding factors were investigated for an association with multiple lesions. Studies that specifically examined risk factors for multiple aneurysms reported a higher proportion of patients with these lesions (Table 3). There was a statistical association between the geographic region and the proportion of patients with multiple intracranial aneurysms (after adjustment for age, $\chi^{2}=$ 298.6, $\mathrm{df}=3, \mathrm{p}<0.001$ ). A higher proportion of multiple intracranial aneurysms was observed in the European, Scandinavian, and US/international studies than in the Japanese studies (Table 3).

Factors that were not statistically associated with multiple intracranial aneurysms include the year of the study, the proportion of patients presenting with a ruptured aneurysm, the proportion of patients who underwent complete angiography, and the proportion of female patients in the study. After adjusting for potential confounding factors, the effect of age remained positive (Table 3). Only three studies that were not already included in the patientlevel analysis provided the mean ages of both the multiple and single aneurysm groups separately. Analysis at the

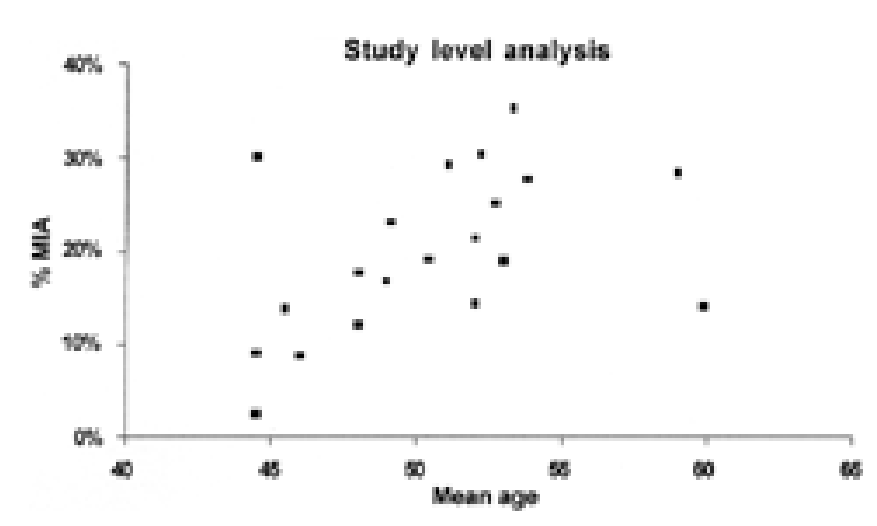

Fig. 1. Scatterplot showing the proportion of patients with at least one aneurysm who have multiple lesions plotted against the mean age (in years) of patients in the study. The studies included in the metaregression are detailed in Table 1 . MIA = multiple intracranial aneurysm. level of multiple compared with single lesions for an association between age and multiple intracranial aneurysms was statistically possible but not attempted.

\section{Patient-Level Analysis}

Five studies provided age stratification of patients with multiple and single lesions. . $^{2,10,15,21,25}$ These studies described 4036 patients with a mean age of 55.8 years, of whom 864 harbored multiple intracranial aneurysms (Fig. 2 ). The study by Juvela ${ }^{13}$ did not report the age-stratified proportion of patients with multiple lesions, but it did provide the OR describing this relationship. This OR was incorporated as a Bayesian informative prior estimate. The effective sample size was therefore increased to 4302 , with multiple aneurysms present in 944 patients. The estimated OR for multiple lesions was 1.011 (95\% CI $1.005-1.018)$. The estimated overall probability of multiple lesions at 55.8 years was $25 \%$ (95\% CI 16-35\%). Allowing for between-study heterogeneity, the $95 \%$ confidence limits for the probability of multiple intracranial aneurysms in individual studies ranged from 11 to $47 \%$. Three of the studies provided data that were further stratified into both age and sex factors. Deriving ORs according to both age and sex was not attempted.

\section{Follow-Up Angiography}

Four studies were identified in which investigators described the proportion of patients in whom de novo aneurysms were discovered on follow-up screening angiography after SAH or identification of an unruptured intracranial aneurysm (Table 4). 1,6,14,37 These studies included 439 patients, of whom 32 had at least one de novo aneurysm after a total of 3807 person-years of follow up. The annual rates of development of de novo aneurysms ranged from a minimum of $0.38 \%$ to a maximum of $1.38 \%$.

\section{Annual Rate of Development of De Novo Aneurysms}

As shown in Fig. 3, according to the study-level an-

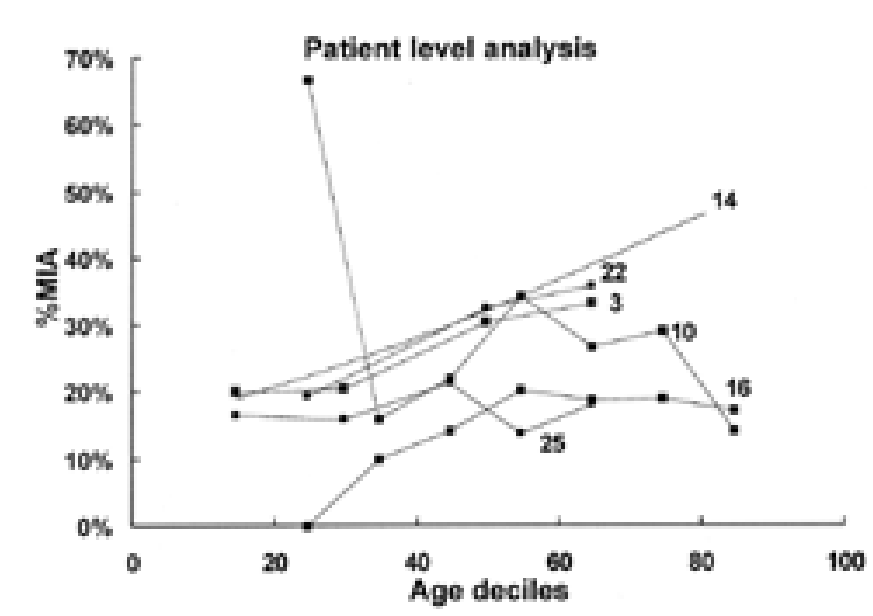

Fig. 2. Graph showing the proportion of patients with at least one aneurysm who were discovered to have multiple lesions, stratified according to age group for five studies. (The study by Juvela did not provide age stratification but did give the OR.) The numbers in the graph correspond to the reference citation for each study. 
TABLE 4

Literature review of follow-up angiography studies in patients with known aneurysms*

\begin{tabular}{|c|c|c|c|c|c|c|c|c|}
\hline Authors \& Year & $\begin{array}{l}\text { Country, Year } \\
\text { Study Began }\end{array}$ & Population & Sample & $\begin{array}{l}\text { Mean FU } \\
(\operatorname{mos})\end{array}$ & $\begin{array}{l}\text { Patients w/ } \\
\text { de Novo } \\
\text { Aneurysms }\end{array}$ & $\begin{array}{c}\text { Annual } \\
\text { Dev Rate } \\
(\%) \dagger\end{array}$ & $\begin{array}{l}\text { Excluded } \\
\text { w/ SAH }\end{array}$ & $\begin{array}{l}\text { Included } \\
\text { w/ UAs }\end{array}$ \\
\hline David, et al., 1999 & US, 1993 & NS & 102 & 4.4 & 6 & 1.38 & NS & 40 \\
\hline Juvela, et al., 2001 & Finland, 1997 & NS & $89 \neq$ & 20.1 & 15 & 0.92 & NS & 10 \\
\hline Tsutsumi, et al., 2001 & Japan, 1985 & 530 & 112 & 9.3 & 9 & 0.90 & 13 & 24 \\
\hline Akyuz, et al., 2004 & Turkey, 1995 & 217 & 136 & 3.9 & 2 & 0.38 & NS & 0 \\
\hline
\end{tabular}

\footnotetext{
* Population refers to the estimated population of patients with aneurysms available for study; sample refers to the number of patients who were alive, healthy, and actually investigated. Abbreviations: dev = development; FU = follow up; UA = unruptured aneurysm.

$\dagger$ The annual rate of development of de novo aneurysms derived from the relationship: proportion with de novo aneurysm $=1-$ $\exp (-$ rate $\times$ years $)$.

$\ddagger$ Of these, 79 had at least two aneurysms at the start of follow up, one treated and one unruptured.
}

alysis, at the mean age of 51.5 years the annual rate of development of de novo aneurysms was $1.62 \%$ (95\% CI $0.28-3.59 \%)$. According to the patient-level analysis, at the mean age of 55.8 years the annual rate of de novo aneurysm development was $0.28 \%$ (95\% CI $0.12-0.49 \%$ ). According to the follow-up studies, the annual rate of de novo aneurysm development was $0.92 \%$ (95\% CI 0.64 $1.25 \%)$.

\section{DISCUSSION}

\section{Risk Factors for Multiple Intracranial Aneurysms}

Using systematic review and metaregression, we estimate that there is a small but significant association between age and development of multiple aneurysms. The estimated association and a simple model of the development of multiple lesions were used to estimate the annual rate of detection of additional lesions in patients who had survived ruptured intracranial aneurysms. The estimated annual rates were small $(0.28-1.62 \%)$. Known or suspected risk factors for multiple intracranial aneurysms include the following: female sex, smoking, hypertension, and a possible family history of intracranial aneurysms or cerebrovascular disease (Table 2).

In this study, we have focused on the relationship between age and multiple aneurysms because it has implications for clinical practice. Providing a complete analysis of the other risk factors was outside the scope of this study. It is likely that there are a number of interacting factors that alter the relationship between age and multiple aneurysms; the prevalence of aneurysms may increase

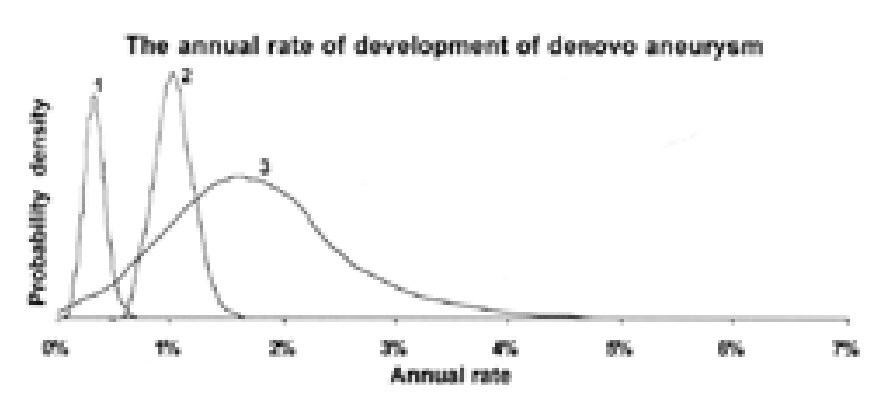

Fig. 3. Plot representing probability density histograms of 30,000 iterations of the Gibbs sampling models. 1 designates a patient-level model, 2 is a follow-up model, and 3 is a study-level model. The absence of a scale on the vertical axis is intentional. with age more quickly in women, smokers, or in those with hypertension, compared with individuals without these risk factors. We did not attempt to estimate the effect of interacting factors because of the limited availability of data.

Earlier studies failed to show a statistically significant association between age and multiple intracranial aneurysms, principally because the association was small. Conducting a very large study or combining the studies with metaregression was necessary to make a more precise estimate. The purpose of our study was not to prove the existence of an association between age and multiple aneurysms. Rather, its purpose was to estimate the size of the association to aid clinical decision making about the management of disease in patients who have survived a ruptured aneurysm. Despite this purpose, we noted an association between multiple intracranial aneurysms and the geographic region in which patients lived, but not the proportion of females.

\section{Study Limitations}

Most of the patients who were included in these studies had a history of ruptured aneurysms. The mean ages of the patients ranged from 44.5 to 60 years. Therefore, the results of this analysis should be generalized to this broad group of patients and will have less relevance to younger patients or those with no history of ruptured aneurysms. In this study we examined the proportion of patients with aneurysms who have multiple lesions. The study should not be generalized to cover the proportion of patients who have three aneurysms among those who already have two.

A bias may arise from the publication of early, small studies that report surprising results or positive treatment effects or if publication was influenced by the results of the study. In most of the studies included under our criteria, we cannot see a plausible relationship between publication and either the association between age and multiple aneurysms or the prevalence of multiple lesions. The higher incidence of multiple intracranial aneurysms in studies in which patients were examined for risk factors for multiple lesions is possibly a result of publication bias. The higher incidence of multiple lesions in these studies may also result from increased investigation, diagnosis, or documentation of multiple aneurysms relative to the remaining studies.

Patient-level analysis was only possible for five of the studies. This is a stronger design than study-level analysis. 
Contacting the investigators may have increased the number of studies with patient-level data but many of these investigations were conducted 10 to 20 years ago. We were unable to confirm the number of patients who underwent complete cerebral angiography in 10 of the studies; indeed in three of the reports patients with incomplete angiography were included. . $^{13,30,31}$ In one study, patients older than 60 years of age were excluded because incomplete angiography was routine for this age group. ${ }^{13}$ We did not identify a statistical association between the study reporting complete cerebral angiography in all patients and the proportion of multiple intracranial aneurysms. This contrasts with the findings of Rinne, et al., ${ }^{31}$ who reported in 1995 that the main factors influencing the reported proportions of multiple lesions were the quality of the angiography, the number of arteries imaged, and the referral policy.

It is possible that the association between age and multiple aneurysms may be biased by the extent of the cerebral angiography performed in older patients in earlier studies. The patient-level analysis included two early studies in which older patients may have been underinvestigated. Although the regression methods provide estimates of the rate of de novo aneurysm development, the follow-up studies have a stronger design. The CIs around a rate depend only on the number of events that have occurred and not the size of the denominator. The number of aneurysms detected in the follow-up studies was small (two-15), indicating low precision. Because of the low rate of developing aneurysms, follow-up studies need to contain large numbers of patients to estimate with precision the annual rate of development.

\section{Major Biases}

Patients with ruptured aneurysms may have a higher proportion of multiple lesions than do patients with unruptured aneurysms. This is because the presence of multiple intracranial aneurysms is likely to be a risk factor for an individual who suffers a hemorrhage compared with those in whom a single lesion is present. We would expect this to cause an overestimation of the rate of de novo aneurysm development by approximately a factor of 1.5 to 2 in the patient-level and study-level analyses.

Patients with ruptured aneurysms who underwent angiography screening in subsequent years represented approximately 10 to $20 \%$ of the population with aneurysm rupture in the follow-up studies (Table 4). These survivors have not died, become disabled, or experienced a recurrent aneurysm rupture. They have been available for follow up and volunteered for the investigation. This may cause an underestimation of the annual rate of de novo aneurysm development derived from the analysis of follow-up studies.

Interestingly, the same factors will influence the likelihood that individuals will undergo angiography in clinical practice. This diminishes the effect of this bias on the generalizability of the results. In one of the follow-up studies, $89 \%$ of the participants had at least two aneurysms at the start of the follow-up period (Table 4). This may cause an overestimation of the annual rate compared with populations with single lesions.

\section{Hierarchical Models}

Hierarchical or random-effect models are well described in the medical literature ${ }^{4,36}$ for both bayesian and frequentist statistical methods but may not be familiar to some readers. A parameter estimated based on a number of different studies will inevitably vary between the studies. Variance between the studies arises from four sources. 1) At the study level there is sampling error that is related to the size of the study. 2) Between the studies there is variance from measurable sources such the mean age of the patients. 3) Between the studies there is variance from nonmeasurable or random factors termed heterogeneity. 4) There is the variance in the estimate of the overall mean for the parameter of interest.

Hierarchical models tend to reduce the sampling error associated with each individual study, provide a flexible tool for metaregression, and can be used to estimate the variance both from random between-study heterogeneity and from uncertainty in the estimate of the overall mean. These models are used to estimate confidence limits around the overall mean of the parameter and wider confidence limits around the mean of predicted future individual studies. The alternative to random-effects models are fixed-effects models that ignore the random between-study factors. Bayesian models have even more flexibility because of their ability to incorporate prior information. In this review only one previously reported OR for the association between age and multiple intracranial aneurysms was available and it was used as a prior estimate for the patient-level analysis. ${ }^{13}$

\section{Constant Annual Rate and the Proportion With De Novo Aneurysms}

Some readers may not be familiar with the exponential survival distribution. This distribution is appropriate when events are assumed to occur at a constant rate with respect to both time and the number of individuals who remain at risk at any point in time. ${ }^{8}$ The proportion "surviving," designated as $S(t)$, without the event occurring after a given time $t$ is calculated as $S(t)=\exp (-$ rate $\times t)$.

The proportion of patients in whom the event occurred after a given time is $1-\mathrm{S}(\mathrm{t})$. In the case of screening for de novo aneurysms, the probability of having at least one de novo lesion will be $1-\exp (-$ rate $\times \mathrm{t})$, where $t$ is the time in years since the last angiogram and the rate is approximately 0.28 to $1.62 \%$. This relationship can be used to derive the rate of aneurysm development from the proportion of patients in whom angiography results are positive when screening is performed. This method should be distinguished from a rate derived from the proportion of patients with multiple intracranial aneurysms divided by the mean duration of observation until a screening test is performed. ${ }^{6,37}$ The proportion of individuals with positive findings of at least one aneurysm on angiographic studies is unlikely to have a linear relationship with the mean time until a screening test is performed.

\section{CONCLUSIONS}

The estimated annual rate of development of second de 
novo aneurysms ranged from 0.28 to $1.62 \%$. These estimated annual rates may be used to predict the probability of aneurysm detection later in life after a lesion ruptures. By taking the time in years since the last angiogram and assuming a constant annual rate, we may use the exponential survival model. Assuming an annual rate of $1 \%$, we may predict probabilities of $4.9 \%$ for de novo aneurysms at 5 years, $9.5 \%$ at 10 years, and $18 \%$ at 20 years. If the decision is made to screen for de novo aneurysms it should not be done frequently. Aneurysms previously treated with coil or clip placement have a recognized rate of recurrence or rebleeding. ${ }^{39}$ The perceived importance of detecting both de novo and recurrent aneurysms will influence the decision to perform angiography after treating one of these lesions.

Is the predicted annual development rate of de novo aneurysms consistent with the prevalence of multiple lesions? Both the prevalence of incidental aneurysms and the incidence of rupture greatly increase with patient age. ${ }^{3,9,23,27}$ Assuming that the first aneurysm develops at a constant rate after the age of 20 years, and assuming that the annual rate of de novo lesions is $1 \%$, the predicted rate of multiple intracranial aneurysms is $9.5 \%$ at 40 years of age, $14 \%$ at 50 years, and $18 \%$ at 60 years. This is comparable to but less than the rate reported in most of the studies reviewed in Table 1 and Figure 2. Therefore, it appears likely that most second aneurysms are "degenerative" and develop at a slow and constant rate as the population with a single aneurysm ages. There is likely to be an additional subgroup of the population in whom congenital multiple lesions develop earlier in adult life. Congenital, genetic, or sex-related risk factors are likely to be associated with this subgroup in which multiple intracranial aneurysms develop earlier in adult life. Screening investigations have been performed to detect asymptomatic familial aneurysms. ${ }^{5,17,19}$ We would expect that the rate of de novo aneurysms detected would be higher in patients with a previous rupture than in those with a positive family history.

\section{Appendix}

We presented a logistic model of the following form:

$\operatorname{logit}\left(\pi_{i}\right)=\beta_{0}+\beta_{1}\left(a g e_{i}-\overline{a g e}\right)+\alpha_{i}$.

Rearranging this equation gives us the study probability:

$\pi_{i}=\exp \left(L_{i}\right) /\left[1+\exp \left(L_{i}\right)\right]$

where $L_{i}=\beta_{0}+\beta_{1}($ age $-\overline{\text { age }})+\alpha_{i}$.

Taking the derivative of the probability with respect to age and dividing by 1 minus the probability gives us the rate:

rate $_{i}=\beta_{1}\left\{\exp \left(L_{i}\right) /\left[1+\exp \left(L_{i}\right)\right]\right\}$

The rate can be rewritten in terms of the probability, giving us the simple relationship rate $_{1}=\beta_{1} \pi_{i}$. The overall rate is derived from the overall probability calculated at the mean age:

$\pi=\exp \left(\beta_{0}\right) /\left[1+\exp \left(\beta_{0}\right)\right]$.

\section{References}

1. Akyuz M, Tuncer R, Yilmaz S, et al: Angiographic follow-up after surgical treatment of intracranial aneurysms. Acta Neurochir 146:245-250, 2004
2. Andrews RJ, Spiegel PK: Intracranial aneurysms. Age, sex, blood pressure, and multiplicity in an unselected series of patients. J Neurosurg 51:27-32, 1979

3. Anonymous: Epidemiology of aneurismal subarachnoid hemorrhage in Australia and New Zealand: incidence and case fatality from the Australasian Cooperative Research on Subarachnoid Hemorrhage Study (ACROSS). Stroke 31:1843-1850, 2000

4. Austin PC, Goel V, van Walraven C: An introduction to multilevel regression models. Can J Public Health 92:150-154, 2001

5. Bromberg JE, Rinkel GJ, Algra A, et al: Familial subarachnoid hemorrhage: Distinctive features and patterns of inheritance. Ann Neurol 38:929-934, 1995

6. David CA, Vishteh AG, Spetzler RF, et al: Late angiographic follow-up review of surgically treated aneurysms. J Neurosurg 91:396-401, 1999

7. Ellamushi HE, Grieve JP, Jager HR, et al: Risk factors for the formation of multiple intracranial aneurysms. J Neurosurg 94: 728-732, 2001

8. Hogg RV, Tanis EA: Probability and Statistical Inference. New York: Macmillan Publishing, 1988, pp 172-182

9. Horikoshi T, Akiyama I, Yamagata Z, et al: Retrospective analysis of the prevalence of asymptomatic cerebral aneurysm in 4518 patients undergoing magnetic resonance angiographywhen does cerebral aneurysm develop? Neurol Med Chir 42: $105-113,2002$

10. Inagawa T: Multiple intracranial aneurysms in elderly patients. Acta Neurochir 106:119-126, 1990

11. International Study of Unruptured Intracranial Aneurysms Investigators: Unruptured intracranial aneurysms - risk of rupture and risks of surgical intervention. N Engl J Med 339: 1725-1733, 1998

12. Jomin M, Lesoin F, Lozes G: Prognosis with 500 ruptured and operated intracranial arterial aneurysms. Surg Neurol 21: 13-18, 1984

13. Juvela S: Risk factors for multiple intracranial aneurysms. Stroke 31:392-397, 2000

14. Juvela S, Puossa K, Porras M: Factors affecting formation and growth of intracranial aneurysms: a long-term follow-up study. Stroke 32:485-491, 2001

15. Kaminogo M, Yonekura M, Shibata S: Incidence and outcome of multiple intracranial aneurysms in a defined population. Stroke 34:16-21, 2003

16. Kassell NF, Torner JC, Haley EC Jr, et al: The International Cooperative Study on the Timing of Aneurysm Surgery. Part 1: Overall management results. J Neurosurg 73:18-36, 1990

17. Leblanc R, Melanson D, Tampieri D, et al: Familial cerebral aneurysms: a study of 13 families. Neurosurgery 37:633-639, 1995

18. Molyneux A, Kerr R, Stratton I: International Subarachnoid Aneurysm Trial (ISAT) of neurosurgical clipping versus endovascular coiling in 2143 patients with ruptured intracranial aneurysms: a randomised trial. Lancet 360:1267-1274, 2002

19. Nakagawa T, Hashi K, Kurokawa Y, et al: Family history of subarachnoid hemorrhage and the incidence of asymptomatic, unruptured cerebral aneurysms. J Neurosurg 91: 391-395, 1999

20. Nakstad P, Nornes H, Hauge HN, et al: Cerebral panangiography in spontaneous subarachnoid hemorrhage from intracranial aneurysms. Acta Radiol 29:633-636, 1988

21. Nishioka H, Torner JC, Graf CJ, et al: Cooperative study of intracranial aneurysms and subarachnoid hemorrhage: a longterm prognostic study. II. Ruptured intracranial aneurysms managed conservatively. Arch Neurol 41:1142-1146, 1984

22. Ogilvy CS, Carter BS: Stratification of outcome for surgically treated unruptured intracranial aneurysms. Neurosurgery 52: 82-88, 2003

23. Ohkuma H, Fujiita S, Suzuki S: Incidence of aneurysmal sub- 
arachnoid hemorrhage in Shimokita, Japan, from 1989 to 1998. Stroke 33:195-199, 2002

24. Orz Y, Kobayashi S, Osawa M, Tanaka Y: Aneurysm size: a prognostic factor for rupture. Br J Neurosurg 11:144-149, 1997

25. Ostergaard JR, Hog E: Incidence of multiple intracranial aneurysms. Influence of arterial hypertension and gender. J Neurosurg 63:49-55, 1985

26. Pasqualin A, Bazzan A, Cavazzani P, et al: Intracranial hematomas following aneurysmal rupture: experience with 309 cases. Surg Neurol 25:6-17, 1986

27. Pobereskin LH: Incidence and outcome of subarachnoid haemorrhage: a retrospective population based study. J Neurol Neurosurg Psychiatry 70:340-343, 2001

28. Pinna G, Pasqualin A, Vivenza C, et al: Rebleeding, ischaemia and hydrocephalus following anti-fibrinolytic treatment for ruptured cerebral aneurysms: a retrospective clinical study. Acta Neurochir 93:77-87, 1988

29. Qureshi AI, Suarez JI, Parekh PD, et al: Risk factors for multiple intracranial aneurysms. Neurosurgery 43:22-27, 1998

30. Rasmussen P, Busch H, Haase J, et al: Intracranial saccular aneurysms. Results of treatment in 851 patients. Acta Neurochir 53:1-17, 1980

31. Rinne J, Hernesniemi J, Niskanen M, Vapalahti M: Management outcome for multiple intracranial aneurysms. Neurosurgery 36:31-38, 1995

32. Rosenorn J, Eskesen V, Schmidt K, et al: Clinical features and outcome in 1076 patients with ruptured intracranial saccular aneurysms: a prospective consecutive study. Br J Neurosurg 1: $33-45,1987$
33. Schievink WI, van der Werf DJ, Hageman LM, et al: Referral pattern of patients with aneurysmal subarachnoid hemorrhage. Surg Neurol 29:367-371, 1988

34. Seifert V, Stolke D, Trost HA: Timing of aneurysm surgery. Comparison of results of early and delayed surgical intervention. Eur Arch Psychiatry Neurol Sci 237:291-297, 1988

35. Stroup DF, Berlin JA, Morton SC, et al: Meta-analysis of observational studies in epidemiology: a proposal for reporting. Meta-analysis Of Observational Studies in Epidemiology (MOOSE) group. JAMA 283:2008-2012, 2000

36. Thompson SG, Turner RM, Warn DE: Multilevel models for meta-analysis, and their application to absolute risk differences. Stat Methods Med Res 10:375-392, 2001

37. Tsutsumi K, Ueki K, Morita A, et al: Risk of aneurysm recurrence in patients with clipped cerebral aneurysms: results of long-term follow-up angiography. Stroke 32:1191-1194, 2001

38. Wiebers DO, Whisnant JP, Huston J III, et al: Unruptured intracranial aneurysms: natural history, clinical outcome, and risks of surgical and endovascular treatment. Lancet 362:103-110, 2003

39. Yamakawa H, Sakai N, Takenaka K, et al: Clinical analysis of recurrent subarachnoid hemorrhage after neck clipping surgery. Neurol Med Chir 37:380-386, 1997

Manuscript received September 15, 2004.

Accepted in final form October 5, 2004.

Address reprint requests to: James van Gelder, M.D., Department of Neurosurgery, Liverpool Hospital, Liverpool, New South Wales 2170, Australia. email: james.gelder@swsahs.nsw.gov.au. 